\title{
Investigation of hydro magnetic steady flow between two infinite parallel vertical porous plates
}

\author{
Kimeu Boniface ${ }^{1}$, Kwanza Jackson ${ }^{2}$, Onyango Thomas ${ }^{3}$ \\ ${ }^{1}$ Mathematics and Computer Science Department, Catholic University of Eastern Africa, Nairobi, Kenya \\ ${ }^{2}$ Pure and Applied Mathematics Department, Jomo Kenyatta University of Agriculture and Technology, Nairobi, Kenya \\ ${ }^{3}$ Industrial and Engineering Mathematics Department, Technical University of Kenya, Nairobi, Kenya

\section{Email address:} \\ mulonzik@gmail.com (K. Boniface),kwanzakioko@yahoo.com (K. Jackson), Onyangottm@gmail.com (O. Thomas)
}

\section{To cite this article:}

Kimeu Boniface, Kwanza Jackson, Onyango Thomas. Investigation of Hydro Magnetic Steady Flow between Two Infinite Parallel Vertical Porous Plates. American Journal of Applied Mathematics. Vol. 2, No. 5, 2014, pp. 170-178. doi: 10.11648/j.ajam.20140205.14

\begin{abstract}
In this study we consider MHD steady fluid flow between two infinite parallel vertical porous plates with heat transfer. The governing equations considered we reduced to specific form according to the geometry of the studied problem. The non-dimensional governing equations involved in the present analysis are solved using the finite difference technique and the expressions for velocity and temperature distributions have been obtained. The effect of different parameters such as magnetic parameter, Prandtl number, thermal Grashoff number, and the temperature and velocity distributions are discussed.
\end{abstract}

Keywords: Magneto Hydrodynamics, Incompressible Fluid, Transverse, Steady State

\section{Introduction}

Fluid is a substance that undergoes deformation when an external force is applied to it however small it may be. Steady motion is one where there is no velocity variation along the direction of flow otherwise it is termed as unsteady. Magneto hydrodynamics as a word was first used by Hannes Alfen in 1942 for which he received a Nobel Prize in physics in 1970. Initially MHD include only the study of strictly incompressible fluids but today it is applied to studies of partially ionized gases as well. Other names have been suggested such as magneto fluid- mechanics, magneto aerodynamics or hydro-magnetics but still the original Nomenclature persists.

Magneto hydrodynamics (MHD) is the study of flow of electrically conducting fluid in the presence of magnetic field. The word magneto hydrodynamic (MHD) is derived from: Magneto-meaning magnetic field, Hydro meaning Liquid and Dynamics which means movement. Hydrodynamics is the study of fluid flow and the forces that cause the flow in the absence of the electromagnetic field. In MHD a current is induced when a current conductor moves in a magnetic field. Hence when a viscous conducting fluid flows in the presence of a transverse magnetic field, electromagnetic forces act on the fluid particles thereby altering the geometry of the motion.

The presence of magnetic fields leads to forces that in turn act on the fluid potentially altering the geometry and the strength of the magnetic fields themselves. MHD phenomena results from the mutual effect of a magnetic field and a conducting fluid flowing across it. Thus electromagnetic force is produced in a fluid flowing across a transverse magnetic field and the resulting current and magnetic fields combine to produce a force that resists the fluid motion. The current also generates its own magnetic field which distorts the original magnetic field and the Science of MHD is a detailed study of these phenomena which occur both in nature and in engineering.

For a long time it has been suspected that most of the matter in the universe is in plasma or highly iionized gaseous state and much of the basic knowledge in the area of electromagnetic fluid dynamics evolved from these studies.

\section{Literature Review}

The concept of MHD is largely perceived to have been initiated by Faraday when he did the first quantitative observation of Magneto hydro dynamics. He did experiments with mercury as a conducting fluid flowing in a glass tube placed in magnetic field and observed that voltage was induced in direction perpendicular to both the direction of flow and magnetic field. He further showed that when an electric field is applied to a conducting fluid in the direction 
which is perpendicular to magnetic field, a force is exerted on the fluid in the direction perpendicular to both electric field and magnetic field. Since then a lot has been done on MHD and its related fields Rao et al (1990) studied the heat transfer in porous medium in the presence of transverse magnetic field. The effects of the heat source parameter and Nusselt number were analyzed. They discovered that the effect of increasing porous parameter is to increase the Nusselt Number.

Kinyanjui et al (2003) investigated MHD Stokes problem for a vertical infinite plate in dissipative rotating fluid with Hall current as Sigey et al (2004) presented an investigation on the numerical study on natural convection turbulent heat transfer in an enclosure. Rajput et al (2011) studied Natural convection in unsteady hydro magnetic couette flow through a vertical channel in the presence of thermal radiation. The effect of different parameters like magnetic parameter, Prandtl number, radiation parameter, thermal Grashof number, accelerating parameter and time on the temperature, velocity, skin-friction and Nusselt were discussed.

Okello et al (2007) investigated, unsteady free convection incompressible fluid past a semi infinite vertical porous plate in the presence of a strong magnetic field inclined at an angle $\alpha$ to the plate with Hall and ion-slip current effects. The effects of modified Grasshof number, suction velocity, the angle of inclination, time, Hall current, ion-slip current, Eckert number, Schmidt number and heat source parameter on the convectively cooled or convectively heated plate restricted to laminar boundary layer were studied. He found that an increase in mass diffusion parameter Sc causes a decrease in concentration profiles, absence of suction velocity or an increase of it causes an increase of concentration profiles, an increase of Eckert number causes an increase in temperature profiles and also an increase of an angle on inclination leads to an increase in primary velocity profiles but a decrease in secondary velocity profiles.

Baoku et al (2010) investigated the problem of hydro magnetic Couette flow of a high viscous fluid through a porous channel in the presence of an applied uniform transverse magnetic field and thermal radiation. Effects of permeability parameter for the cases of low, moderate and high permeabilities on the numerical solutions were obtained for different magnetic parameters and Nahme number. Temperature and velocity profiles are presented for different Nahme and magnetic field parameters to reveal the coupled effects of thermal radiation and magnetic field were shown. They concluded that the increase in thermal radiation of the fluid results to a decrease in the temperature profiles of the hydro magnetic Couette fluid, the permeability of the porous medium and thermal radiation have insignificant effects on the steady hydro magnetic Couette fluid flow and that the increase in magnetic field leads to an increase in the velocity profiles with significant effects of low and moderate permeability parameters except at high medium permeability with very high magnetic field where increase in magnetic

Okwoyo J. M. and Sing C. B. (2008) presented a paper on steady laminar flow of viscous incompressible fluid between two parallel infinite plates when upper plate is moving with constant velocity and lower plate is held stationary under the influence of transverse magnetic field. The resulting expression was solved by the application of Laplace transform and analytical expression was obtained. Sigey et al (2012) carried out a study of magnetic hydrodynamic free convective flow past an infinite vertical porous plate in an incompressible electrically conducting fluid. The investigation of the effect of viscous dissipation on the velocity profiles and temperature distribution of the fluid in the presence of a transverse magnetic field subject to a constant suction velocity was conducted. The partial differential equations governing the flows were analyzed using an explicit finite difference method. The numerical results of the study showed that an increase in the viscous dissipation causes an increase in the velocity profiles and temperature distribution of the fluid. This study finally asserted that an increase in the viscous dissipation parameter or term leads to an increase in velocity and temperature profiles. This increase in the velocity profiles and temperature profile occurred at a distance away from the porous plate.

A study of magnetic hydrodynamic free convective flow past an infinite vertical porous plate in an incompressible electrically conducting fluid was considered. The numerical results of the study shown that an increase in the Grasshof number causes an increase in the velocity profiles; an increase of Hartman number causes a decrease of velocity profile whereas an increase of Prandtl number causes a decrease in temperature distribution (Amenya et al, 2013).

Nyabuto et al (2013) studied a steady MHD stokes free convection flow of an incompressible, electrically conducting fluid between two parallel infinite plates subjected to constant heat flux and pressure gradient. They obtained results on velocity profiles and temperature distribution and discussed in detail. An increase in Hartmann is found to cause a decrease in velocity profiles and an increase in temperature distribution. In addition, an increase in Eckert number causes an increase in temperature while an increase in Prandtl leads to a fall in temperature distribution. Their results were found to merge with the physical situation of the flow. In this study we consider MHD steady fluid flow between two infinite parallel vertical porous plates with heat transfer.

\section{Governing Equations}

MHD governing equations results from a combination of two disciplines namely the electromagnetic theory and fluid mechanics. The basic equations of MHD for incompressible fluids can therefore be written as the Maxwell's equation, Ohm's law, the equation of continuity and the equation of motion. If the fluid is in motion the velocity may be different at each location in the fluid.

\section{Maxwell's Equations}

This is a set of four equations that describes the relationships between electric and magnetic fields and their source. In different form they are; 


$$
\begin{aligned}
& \nabla \times E=-\frac{\partial \vec{B}}{\partial t} \text { (Faradays law of induction) where } \vec{B}=\mu_{e} \vec{H} \\
& \nabla \cdot B=0 \quad \text { (Gauss law of magnetism) } \\
& \nabla \cdot D=\rho_{e} \text { Or } \nabla \cdot \vec{E}=\frac{P_{e}}{\rho} \quad \text { (Gauss law) } \\
& \nabla \times \vec{B}=\mu_{e} \vec{J}+\mu_{e} \frac{\partial \vec{D}}{\partial t} \quad \text { (Amperes law) }
\end{aligned}
$$

The Ohm's law

The ohms law is $\vec{J}=\sigma(\vec{E}+\vec{V} \times \vec{B})$

The equation of continuity

This equation expresses mathematically the law of conservation of mass. According to this law mass can neither be created nor destroyed. Raisinghai (2006) showed that the continuity equation gives the fact that increase in the mass of the fluid within any closed surface drawn in the fluid at any time must equal to the excess of mass that flows out.

The equation in vector form is;

$$
\frac{\partial \rho}{\partial t}+\vec{\nabla} \cdot(\rho \vec{q})=0
$$

For any incompressible fluid in which density is constant the equation in Cartesian coordinates becomes;

$$
\frac{\partial u}{\partial x}+\frac{\partial v}{\partial y}+\frac{\partial w}{\partial z}=0
$$

\section{Equation of Momentum}

The equation of momentum is also called the equation of motion. The law of conservation of momentum states that initial momentum is equal to the final momentum. Using Newton's second law of motion which states that the net force acting on a body is equal to the product of mass and acceleration and applying this on an infinitesimal fluid element the Navier stokes equation for an incompressible, viscous fluid becomes;

$$
\rho \frac{\partial \vec{V}}{\partial t}+\rho(\vec{V} \cdot \nabla) \vec{V}=-\nabla P+v \nabla^{2} \vec{V}+\rho F_{b}
$$

From Faraday's law that states that if current is passing through a conductor under a magnetic flux then the conductor experiences a force perpendicular to both of them and is proportional to the product of their magnitude. Hence, force acting on the conductor is

$$
\vec{F}_{e}=\vec{J} \times \vec{B}
$$

This force is called the Lorentz's force or electromagnetic force, hence the equation can be written as

$$
\rho \frac{\partial \vec{V}}{\partial t}+\rho(\vec{V} . \nabla) \vec{V}=-\nabla P+v \nabla^{2} \vec{V}+\rho F_{b}+\rho(\vec{J} \times \vec{B})
$$

Since this is a two dimensional flow the equation describing the motion are reduced to;

$$
\begin{gathered}
\rho\left[\frac{\partial u}{\partial t}+u \frac{\partial u}{\partial x}+v \frac{\partial v}{\partial y}\right]=\rho F_{x}-\frac{\partial P}{\partial x}+\mu\left[\frac{\partial^{2} u}{\partial x^{2}}+\frac{\partial^{2} u}{\partial y^{2}}\right]+\rho(\vec{J} \times \vec{B}) \\
\rho\left[\frac{\partial v}{\partial t}+u \frac{\partial v}{\partial x}+v \frac{\partial v}{\partial y}\right]=\rho F_{y}-\frac{\partial P}{\partial y}+\mu\left[\frac{\partial^{2} v}{\partial x^{2}}+\frac{\partial^{2} v}{\partial y^{2}}\right]+\rho(\vec{J} \times \vec{B})
\end{gathered}
$$

\subsection{Geometry of the Problem}

In this study we consider the steady, two dimensional laminar free convective flow of an electrically conducting viscous incompressible fluid between two infinite parallel porous plates with a transverse magnetic field applied as shown in the figure, $\mathrm{x}$-axis is taken between the plates parallel to each and in the vertically upward direction and y-axis is taken normal to the plates. A magnetic field of uniform strength is applied transversely to the direction of flow. Since the plates are infinite in length all variables are functions of $y$ only. The assumption is that the velocity and magnetic distribution vectors are respectively of the form.

$$
\begin{gathered}
\vec{V}=\vec{V}[u, v, 0] \\
\vec{H}_{0}=\left[H_{x}(y), H_{0}, 0\right]
\end{gathered}
$$

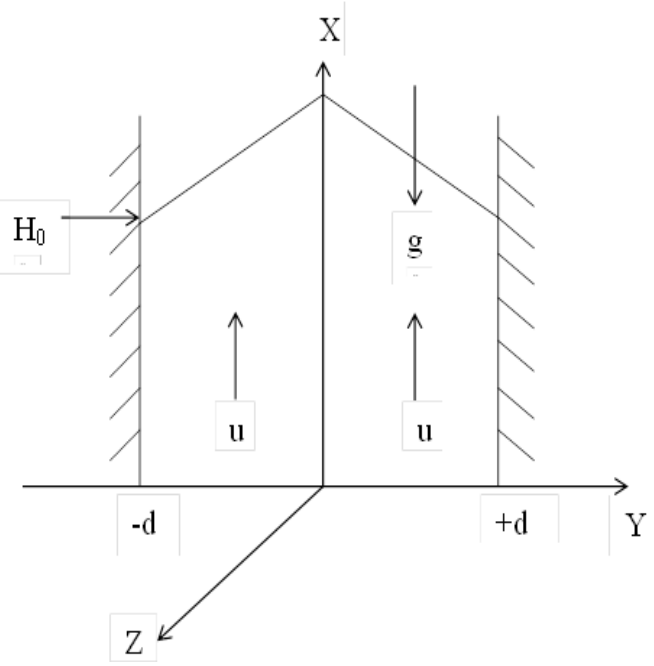

Figure 1.1. Geometry of the research problem

\subsection{Specific Approximations and Assumptions of this Study}

We take the origin at the lower edge of the plates, $\mathrm{x}$-axis along the plates and the y-axis normal to the plates. We also make the following approximations and assumptions.

1. The fluid is finitely conducting. This makes the viscous dissipation and joules heating negligible but induced magnetic field is not negligible.

2. Body forces caused by gravity and magnetic field are important in this study

3. There is no variation of flow and heat transfer quantities in the z-direction 
4. Hall effects and polarization effects are negligible

5. The density is constant except in its contribution to the buoyant forces

6. The plates are electrically non-conducting

7. The plates are infinite in length and hence all variable are functions of $y$

8. The magnetic Reynolds number is small

\subsection{MHD Dimensional Parameters}

Fluid motion is governed by various forces. Inertia force (ability to resist change) always exists in all flow problems. By considering the ratio of inertia force to any other force governing the particular flow such as viscous force, gravitational force, pressure force or magnetic force we obtain some dimensionless parameters. The following dimensionless parameters will be very useful.

\subsection{The Magnetic Pressure Number $\left(R_{H}\right)$}

This is the ratio of the magnetic pressure to the dynamic pressure. Hence $R_{H}=\frac{\mu_{e} H_{0}^{2}}{\rho u^{2}}=\frac{V_{H}^{2}}{u^{2}}=\frac{1}{m_{m}^{2}}$ where $V_{H}=H_{0} \sqrt{\frac{\mu_{e}}{\rho}}$ and is the speed of Alfven's wave and $M_{m}=\frac{U}{V_{H}}$ is the magnetic mach number. For $R_{H}<1$ the terms due to the magnetic field in the equation of motion may be neglected and the fluid motion may not be affected noticeably by the magnetic field. For $R_{H} \geq 1$ the fluid flow will be affected greatly by the magnetic field. Also the flow pattern in MHD may be different when $M_{m}$ is greater or less than unity, usually referred to a super-Alfven or sub-Alfven flow respectively.

\subsection{The Prandtl Number $\left(P_{r}\right)$}

This is the ratio of viscous force to the thermal force and is defined as $P_{r}=\frac{\mu C_{p}}{K}$. It shows the relative importance of viscous dissipation to the thermal dissipation.

\subsection{The Magnetic Prandtl Number $\left(P_{m}\right)$}

This is the ratio of vorticity diffusion to magnetic diffusion and defined as $P_{m}=\frac{R_{\sigma}}{R_{e}}=\frac{v}{V_{H}}=\frac{\mu}{\rho} \mu_{e} \sigma$. It is similar to Prandtl number $\left(\mathrm{P}_{\mathrm{r}}\right)$. Magnetic Prandtl number $\left(\mathrm{P}_{\mathrm{m}}\right)$ depends on the properties of the fluid and is a measure of the relative magnitude of the thickness of the boundary layer to that of the magnetic boundary layer. It is usually very small for most conducting fluids but has a significant influence in the engineering use of MHD and the experimental study of the flow problems of MHD in the laboratory

\subsection{The Magnetic Number $\left(R_{m}\right)$}

This is the ratio of magnetic force to the inertial forces. Hence $R_{m}=B\left[\frac{\sigma L}{\rho u}\right]^{\frac{1}{2}}=\sqrt{R_{H} R_{\sigma}}$ If the magnetic Reynolds number is very large, we usually use $R_{H}$ to show the effect on the flow field by the magnetic field. For small $R_{\sigma}$ we use $R_{m}$ to show the electromagnetic effects on the flow field.

\subsection{The Grashoff Number $\left(G_{r}\right)$}

This is the ratio of buoyant forces to the viscous forces. It is defined a; $G_{r}=\frac{g \beta \Delta T L^{3}}{v^{2}}$. The larger the Grashoff number the stronger the convective current. If buoyant forces are neglected it is absent in that particular problem. For $G_{r}>0$, the plate is cooling and for $G_{r}<0$, the plate is getting heated. It is a measure of the thermal equivalence of kinetic energy of the flow to the imposed temperature difference and arises from the inclusion of the viscous dissipation term in the energy equation. Hence is absent when viscous dissipation is neglected

\subsection{Final Set of Governing Equations}

1. Continuity Equation (Mass conservation)

Using the assumptions and approximations as discussed above, we can reduce the general equations of continuity, momentum and induction. The flow is assumed to be moving upwards with a constant velocity U. For a steady and incompressible fluid flow the equation of continuity reduces to;

$$
\frac{\partial u}{\partial x}+\frac{\partial v}{\partial y}+\frac{\partial w}{\partial z}=0
$$

But, all the variables depend on y. Therefore,

$$
\frac{\partial u}{\partial x}=\frac{\partial w}{\partial z}=0
$$

Giving that

$$
\frac{\partial v}{\partial y}=0
$$

On integrating this equation we have;

$$
V(y)=-V_{0}
$$

2. Momentum equation

Considering the momentum equation with Lorentz force we have;

$$
\rho \frac{\partial \vec{V}}{\partial t}+\rho(\vec{V} . \nabla) \vec{V}=-\nabla P+v \nabla^{2} \vec{V}+\rho F_{b}+\rho(\vec{J} \times \vec{B})
$$

Where $\vec{B}$ is the magnetic flux and is given as $\vec{B}=\mu_{e} H$ For a steady and incompressible fluid flow and from the reduced continuity equation $\frac{\partial u}{\partial x}=0$ the left hand side of this equation reduces to

$$
=-V_{0} \frac{\partial u}{\partial y}
$$

The pressure gradient in the $\mathrm{x}$-direction results from the 
change in the elevation of the fluid. Hence;

$$
\rho F_{b}-\nabla P=-\rho g+\rho_{\infty}=\rho\left(\rho_{\infty}-\rho\right)
$$

Using the volumetric coefficient of thermal expansion defined by $\beta=-\frac{1}{\rho}\left[\frac{\Delta \rho}{\Delta T}\right]$ the equation (12) gives;

$$
\rho F_{b}-\nabla P=g \beta \rho\left(T-T_{\infty}\right)
$$

Again, the term $\mu \nabla^{2} \vec{V}$ reduces to

$$
\mu \nabla^{2} \vec{V}=v \frac{\partial^{2} u}{\partial y^{2}}
$$

And by similarity, the term $\nabla^{2} T=\left[\frac{\partial^{2}}{\partial x^{2}}+\frac{\partial^{2}}{\partial y^{2}}\right] T(y)$ reduces to

$$
\nabla^{2} T=\frac{\partial^{2} T}{\partial y^{2}}
$$

Also, the term $\nabla^{2} \vec{H}_{0}=\left[\frac{\partial^{2}}{\partial x^{2}}+\frac{\partial^{2}}{\partial y^{2}}\right] H_{x}(y)$ reduces to

$$
\nabla^{2} \vec{H}_{0}=\frac{\partial^{2} H_{x}}{\partial y^{2}}
$$

Also, the term $\vec{J} \times \vec{B}$ is reduced as follows;

$$
\begin{aligned}
\vec{J} \times \vec{B} & =\vec{J} \times \mu_{e} H \\
& =\left|\begin{array}{ccc}
i & j & k \\
0 & 0 & \frac{-\partial H_{x}}{\partial y} \\
\mu_{e} H_{x} & \mu_{e} H_{0} & 0
\end{array}\right|=i\left[0+H_{0} \frac{\partial H_{x}}{\partial y}\right]-j \mu_{e}\left[H_{x} \frac{\partial H_{x}}{\partial y}\right]+k(0) \\
& =i \mu_{e} H_{0} \frac{\partial H_{x}}{\partial y}+j(0)+k(0) \\
& =i \mu_{e} H_{0} \frac{\partial H_{x}}{\partial y}
\end{aligned}
$$

Now, making use of equations (11), (13), (14), and (21) the momentum equation becomes;

$$
-V_{0} \frac{\partial u}{\partial y}=v \frac{\partial^{2} u}{\partial y^{2}}+\mu_{e} H_{0} \frac{\partial H_{x}}{\partial y}+g \beta \rho\left(T-T_{\infty}\right)
$$

Making use of equations (9) and (17) the induction equation becomes;

$$
V_{0} \frac{\partial H_{x}}{\partial y}=H_{0} \frac{\partial u}{\partial y}+\frac{1}{\sigma \mu_{e}} \frac{\partial^{2} H_{x}}{\partial y^{2}}
$$

Making use of equations (9) and (15) the energy equation becomes;

$$
-V_{0} \frac{\partial T}{\partial y}=\frac{K}{\rho C_{p}} \cdot \frac{\partial^{2} T}{\partial y^{2}}
$$

These equations are solved under the following boundary conditions

$$
\begin{array}{ll}
y=-d & u(-d)=0 H_{x}(-d)=0 T(-d)=T_{w} \\
y=+d & u(+d)=0 H_{x}(+d)=0 T(+d)=T_{\infty}
\end{array}
$$

\subsection{Method of Solution and Discussion of Results}

\subsubsection{Non-Dimensionalization}

It is paramount convenient and more informative to work with dimensionless derivatives such that we can alter the boundary conditions for experimental purpose. In this case the following non-dimensional parameters are used to non-dimensionalize the final set of the governing equations.

$$
\eta=\frac{\gamma}{L}
$$

Where $L$ is the characteristic length

$$
\begin{gathered}
\bar{U}=\frac{U L}{v} \quad \overline{V_{0}}=\frac{\overline{V_{0}} L}{v} \\
\bar{H}_{x}=\frac{L}{v} \sqrt{\frac{\mu_{e}}{\rho} H_{x}} \quad M=\frac{H_{0} L}{v} \sqrt{\frac{\mu_{e}}{\rho}}
\end{gathered}
$$

Using the above non-dimensional parameters, the specific governing equations become;

$\frac{-\bar{V}_{0} v^{2}}{L^{3}} \frac{\partial \bar{u}}{\partial \eta}=\frac{v^{2}}{L^{3}} \frac{\partial^{2} \bar{u}}{\partial \eta^{2}}+\frac{\mu_{e}}{\rho} H_{0} \frac{v}{L^{2}} \sqrt{\frac{\rho}{\mu_{e}}} \cdot \frac{\partial \bar{H}_{x}}{\partial \eta}+g \beta \theta\left(T_{w}-T_{\infty}\right)$

Multiplying through by $\frac{L^{3}}{v^{2}}$ the equation becomes;

$$
-\bar{V}_{0} \frac{\partial \bar{u}}{\partial \eta}=\frac{\partial^{2} \bar{u}}{\partial \eta^{2}}+\frac{\mu_{e}}{\rho} H_{0} \frac{L}{v} \sqrt{\frac{\rho}{\mu_{e}}} \cdot \frac{\partial \bar{H}_{x}}{\partial \eta}+\frac{g \beta \theta\left(T_{w}-T_{\infty}\right)}{v} L^{3}
$$

Dropping the dashes and re-writing this equation we have;

$$
V_{0} \frac{\partial u}{\partial \eta}+\frac{\partial^{2} u}{\partial \eta^{2}}+M \frac{\partial H_{x}}{\partial \eta}+G_{r} \theta=0
$$

Magnetic induction equation becomes;

$$
\frac{-\overline{V_{0}} v^{2}}{L^{3}} \sqrt{\frac{\rho}{\mu_{e}}} \cdot \frac{\partial \bar{H}_{x}}{\partial \eta}=H_{0} \frac{v}{L^{2}} \frac{\partial \bar{u}}{\partial \eta}+\frac{1}{\sigma \mu_{e}} \frac{v}{L^{3}} \sqrt{\frac{\rho}{\mu_{e}}} \cdot \frac{\partial^{2} \bar{H}_{x}}{\partial \eta^{2}}
$$

Dividing through by $\frac{v^{2}}{L^{3}} \sqrt{\frac{\rho}{\mu_{e}}}$ and dropping the dashes we have;

$$
-\bar{V}_{0} \frac{\partial \bar{H}_{x}}{\partial \eta}=M \frac{\partial \bar{u}}{\partial \eta}+\frac{1}{P_{m}} \frac{\partial^{2} \bar{H}_{x}}{\partial \eta^{2}}
$$

Hence we obtain; 


$$
-V_{0} \frac{\partial H_{x}}{\partial \eta}=M \frac{\partial u}{\partial \eta}+\frac{1}{P_{m}} \frac{\partial^{2} H_{x}}{\partial \eta^{2}}
$$

Re-arranging

$$
\frac{\partial^{2} H_{x}}{\partial \eta^{2}}+V_{0} P_{m} \frac{\partial H_{x}}{\partial \eta}+P_{m} M \frac{\partial u}{\partial \eta}=0
$$

The non dimensionalzed energy equation becomes;

$$
-V_{0} \frac{\partial T}{\partial y}=\frac{K}{\rho C_{p}} \cdot \frac{\partial^{2} T}{\partial y^{2}}
$$

Applying the non-dimensional parameters, the energy equation becomes;

$$
-V_{0} \frac{d \theta}{d \eta}=\frac{1}{P_{r}}-\frac{d^{2} \theta}{d \eta^{2}}
$$

Rearranging the energy equation, it becomes;

$$
\frac{d^{2} \theta}{d \eta^{2}}+V_{0} P_{r} \frac{d \theta}{d \eta}=0
$$

Hence the three equations on non-dimensionalzed equations are;

\subsubsection{The Momentum Equation}

$$
\frac{\partial^{2} u}{\partial \eta^{2}}+V_{0} \frac{\partial u}{\partial \eta}+M \frac{\partial H_{x}}{\partial \eta}+G_{r} \theta=0
$$

\subsubsection{The Magnetic Induction Equation}

$$
\frac{\partial^{2} H_{x}}{\partial \eta^{2}}+V_{0} P_{m} \frac{\partial H_{x}}{\partial \eta}+P_{m} M \frac{\partial u}{\partial \eta}=0
$$

\subsubsection{The Energy Equation}

$$
\frac{d^{2} \theta}{d \eta^{2}}+V_{0} P_{r} \frac{d \theta}{d \eta}=0
$$

The equations (35), (36) and (37) were solved using the following dimensionless boundary conditions;

$$
\begin{aligned}
& U(-1)=0 \quad \theta(-1)=1 H_{x}(-1)=0 \\
& U(+1)=0 \quad \theta(+1)=0 H_{x}(+1)=0
\end{aligned}
$$

These equations are solved by the method of linear ordinary differential equations and the results plotted by use of a MATLAB programming application.

\section{Results and Discussions}

To analyze the effects of various flow parameters on velocity and induced magnetic field distributions we maintain all the values of other parameters constant and then vary the concerned parameter. The figures herein show the effects of those parameters.

Fig 4.1 shows the temperature distribution for various values of the suction parameter when other parameters are held constant. From the graph as suction parameter increases the temperature of the fluid decreases.

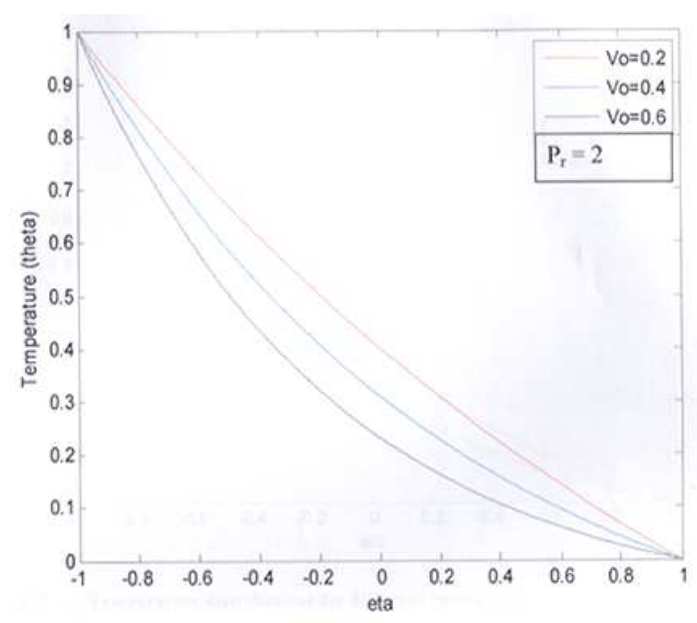

Fig 4.1. Temperature distributions for different values of suction parameter

Fig 4.2 shows that as the Prandtl number increases temperature of the fluid decreases. In heat transfer problems Prandtl number controls the relative thickness of the momentum and thermal boundary layers. When Prandtl number is small as for liquid metals it means that the heat diffuses very quickly and the thermal boundary layer is much bigger than the velocity boundary layer.

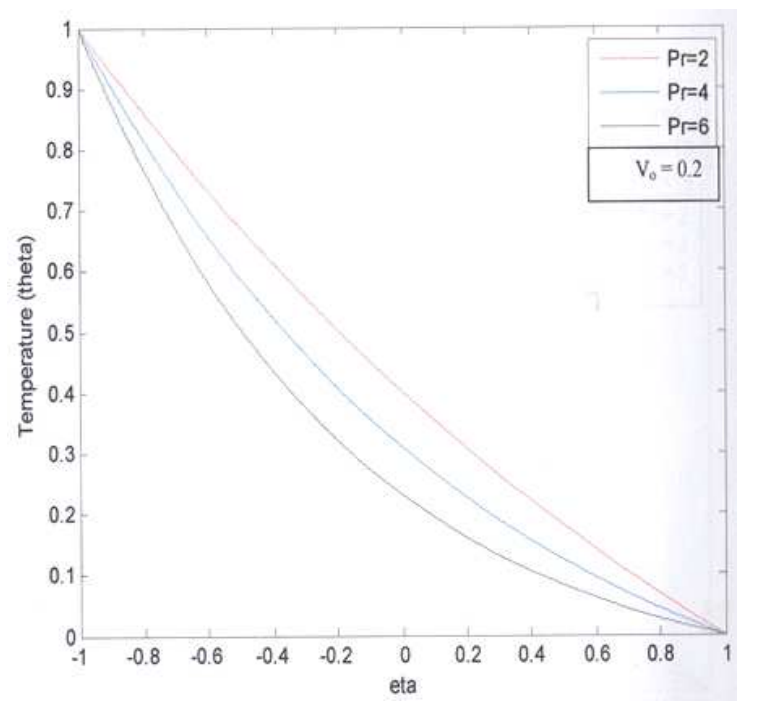

Fig 4.2. Temperature distributions for different values of $\mathrm{Pr}$

Fig 4.3 shows as the Grashoff number increases the velocity increases. Grashoff number gives the ratio of buoyancy forces to the viscous forces. When the Grashoff number increases velocity increases too showing the buoyancy forces are more significant in this flow 


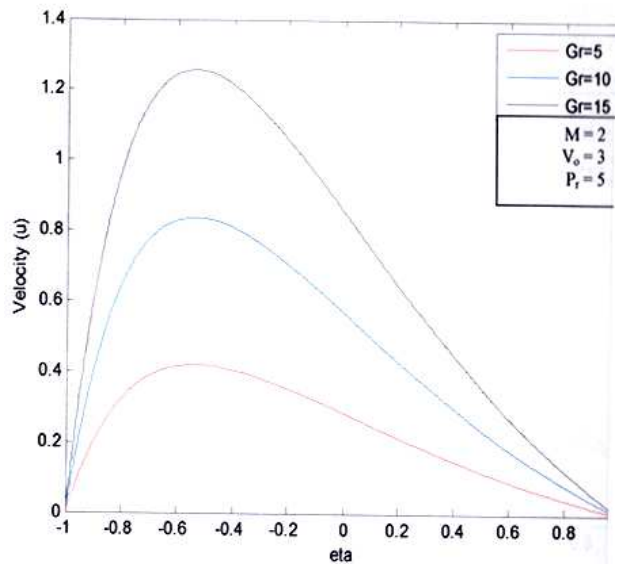

Fig 4.3. Velocity distributions for different values of $G r$

Fig 4.4 shows that as the magnetic field parameter increases the velocity also increases. This shows that the magnetic field strength cannot be neglected such study.

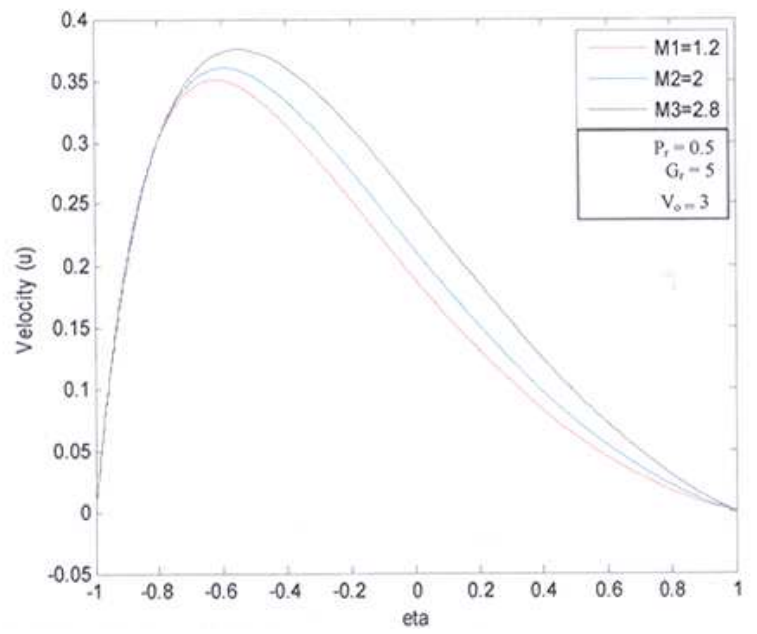

Fig 4.4. Velocity distribution for different values of $M$

Fig 4.5 shows that the as the Prandtl number decreases the velocity increases. Decrease in Prandtl number means the viscous forces are decreasing as the thermal forces increase hence increasing the velocity of the fluid particles.

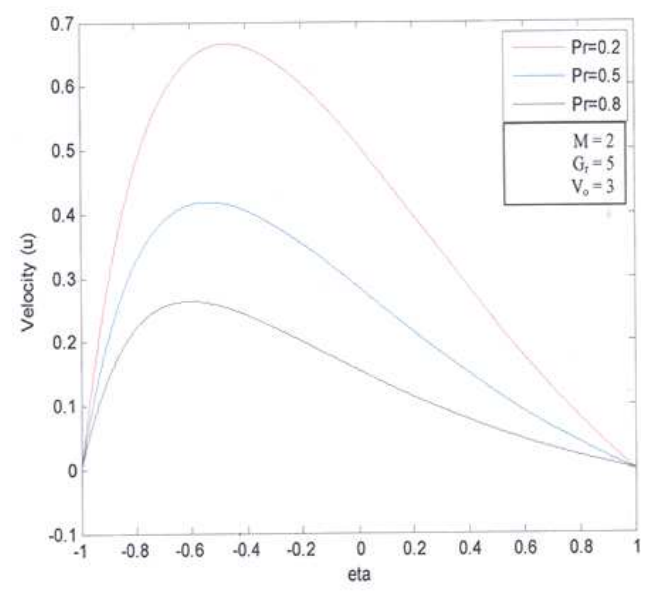

Fig 4.5. Velocity distribution for different values of $\mathrm{Pr}$

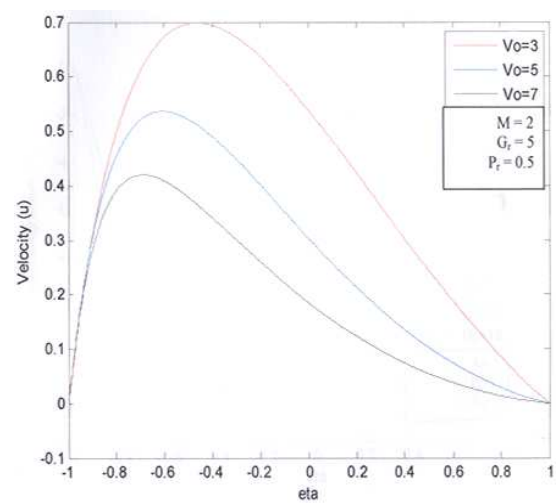

Fig 4.6. Velocity distributions for different values of $V_{o}$

Fig 4.6 shows that as the suction velocity parameter increases the velocity decreases. This shows the suction velocity parameter cannot be neglected in this case since it has effects on the velocity distribution between the plates.

Fig 4.7 shows that the amplitude of the wave increases as the Grashoff number increases. This shows increase in the strength of magnetic field.

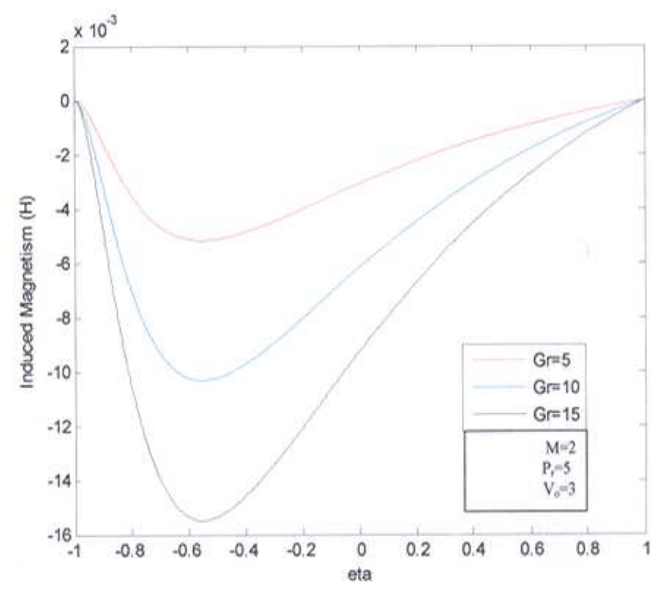

Fig 4.7. Induced magnetic field distributions for different values of Gr

Fig 4.8 shows that as the magnetic field parameter increases the amplitude of the field lines also increases hence an increase in the strength of the magnetic field.

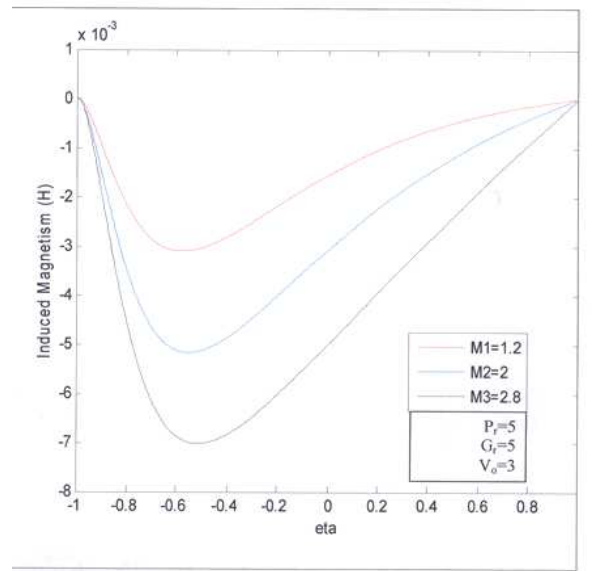

Fig 4.8. Induced magnetic field distribution for different values of $M$ 
Fig 4.9 shows that a decrease in Prandtl number leads to the increase in the amplitude of the magnetic field lines. This shows an increase in the viscous forces in the fluid leads to an increase in the strength of the magnetic field.

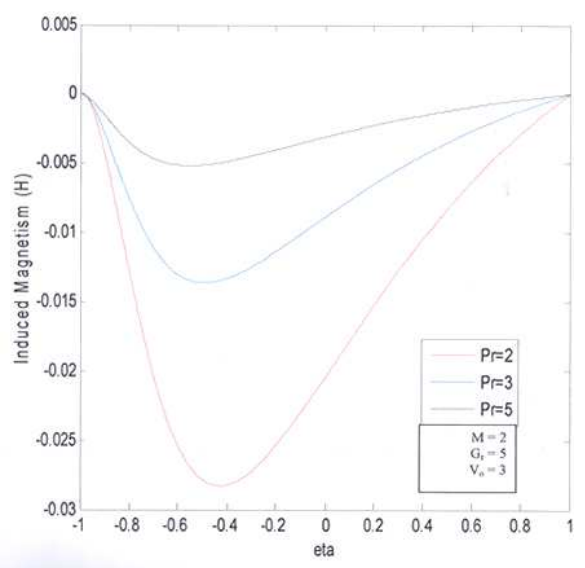

Fig 4.9. Induced magnetic field distribution for different values of Pr

Fig 4.10 shows that as the suction parameter increases the amplitude of the magnetic field lines increases and shifts towards the cooler plate showing the induced magnetic field is stronger nearer the cooler plate where buoyancy forces are less.

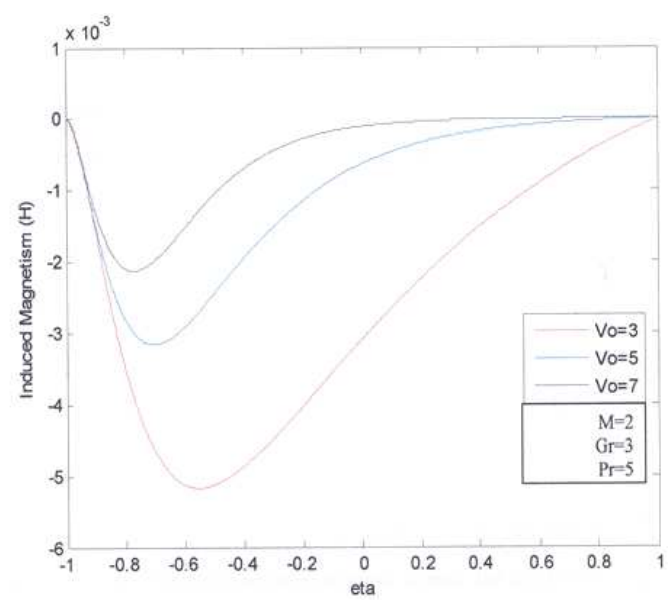

Fig 4.10. Induced magnetic field distribution for different values of $V_{o}$

From Fig 4.7, Fig 4.8, Fig 4.9 and Fig 4.10 it is also found that the lines of force are in a direction opposite to the fluid flow that is with the gravitational force.

\section{Conclusions}

In this study the hydro magnetic steady flow between two parallel porous plates and heat transfer in the presence of a strong magnetic field applied transverse to the direction of flow is considered. The plates were at different temperatures and infinite in length and to diminish the natural free convection which would otherwise occur intensively on the vertical heated plate, the plates are porous so as to maintain the temperature a constant.
It is concluded that increasing the Prandtl number and the suction parameter, decrease in fluid temperature is evident. Increasing the Grashoff number and the magnetic field parameter leads to increase in velocity whereas increase in the Prandtl number and suction velocity decrease the velocity. On the magnetic field parameter and the suction parameter when increased, this leads to the increase in the amplitude of the magnetic field lines.

\section{Nomenclature}

$L \quad$ Characteristic length

$\vec{H} \quad$ Applied magnetic field

$H_{0} \quad$ Intensity of applied magnetic field

$H_{x} \quad$ Induced magnetic field in the x-direction

$\vec{B} \quad$ Magnetic flux field

$C_{v} \quad$ Specific heat at constant volume

$C_{p} \quad$ Specific heat at constant pressure

$G_{r} \quad$ Grashoff number

$M \quad$ Magnetic field parameter

$P_{r} \quad$ Prandtl number

$P \quad$ Pressure

$T \quad$ Absolute Temperature

$V_{0} \quad$ Suction velocity

$\vec{J} \quad$ Current density

$\vec{E} \quad$ Electric field

$P_{m} \quad$ Magnetic Prandtl number

$\vec{V} \quad$ Velocity vector

$\theta \quad$ Non-dimensional temperature

$\mu_{e} \quad$ Magnetic permeability

$\beta \quad$ Coefficient of thermal expansion

$\mu \quad$ Dynamic viscosity

$\rho \quad$ Density

\section{References}

[1] I. G. Baoku, C. Israel-Cookey and B. I. Olajuwon, "Magnetic Field And Thermal Radiation Effects on Steady Hydromagnetic Couette Flow Through A Porous Channel," ISSN. Nigeria, vol. 5, pp. 215-228, 2010.

[2] U. S. Rajput and P. K. Sahu, "Natural Convection In Unsteady Hydromagnetic Couette Flow Througha Verticalchannel In The Presence Of Thermal Radiation", Int. J. of Appl. Math and Mech. India, 2012, pp.35-56.

[3] R. O. Amenya, J. K. Sigey, J. A. Okelo, J. M. Okwoyo, "MHD Free Convection Flow past a Vertical Infinite Porous Plate in the Presence of Transverse Magnetic Field with Constant Heat Flux," IJSR, vol. 2,October 2013.

[4] Rao V. V. and Lingaraj T. (1990) Hall Effect in the viscous incompressible flow through a rotating between two porous walls

[5] Kinyanjui M. Kwanza J.K. Uppal S.M. (2003). MHD stokes free convection flow past an infinite vertical plate subjected to constant heat flux with ion slip current and radiation absorption. Far East Journal of applied mathematics 12 pp105-131. 
[6] Sigey J. Kinyanjui M. and Gatheri J (2004). A numerical study on free convection turbulent heat transfer in an enclosure. JKUAT KENYA

[7] M. D. Raisinghania, (2006) "Kinematics of fluids in Motion. In Fluid Dynamics with complete Hydrodynamics and boundary layer Theory," ( $7^{\text {th }}$ ed., p.29). New Delhi: S. Chand \& Company Ltd.

[8] Okelo J.A (2007). Unsteady free convection incompressible fluid past a semi infinite vertical porous plate in the presence of a strong magnetic fluid inclined at an angle $\alpha$ to the late with Hall and ion slip currents effects JKUAT, Kenya.

[9] Okwoyo J.M. and Sing C. B. (2008) Couette flow between two parallel infinite plates in the presence of transverse magnetic field, J. Kenya Metrological society 2[2], 99 90-94, University of Nairobi Kenya

[10] Nyabuto R., Sigey J.K., Okelo J.A. \& Okwoyo J.M. (2013) Magneto-Hydrodynamics Analysis of Free Convection Flow between Two Horizontal Parallel Infinite Plates Subjected to Constant Heat Flux. The Standard International Journals, Vol. 1, No. 4.

[11] Sigey J. Nyundo S, Gatheri K. F, (2012) Magneto hydrodynamic free convective flow past an infinite vertical porous plate in an incompressible electrically conducting fluid, scientific research 2012 